\title{
Pharmacist-structured review of proton pump inhibitor utilisation in primary care: A non- randomised control study
}

\author{
Su Li Wong, Norharlina binti Sulaiman, Kar Mun Ng, Zhe Yen Lee \\ Wong SL, Norharlina S, Ng KM, et al. Pharmacist-structured review of proton pump inhibitor utilisation in primary care: A non-randomised control \\ study.Malays Fam Physician. 2021;16(3);87-96. https://doi.org/10.51866/oa1153
}

\section{Keywords:}

PPls, drug utilisation review,

pharmacist intervention,

primary care

\section{Authors:}

\begin{abstract}
Introduction: In the primary care setting, proton pump inhibitor (PPI) overutilisation often stems from the failure to discontinue prophylaxis treatment prior to tertiary care discharge and consider step-down therapy following discharge. Long-term PPI use can result in potential drug-related problems and unnecessary drug expenditure. This study aimed to evaluate the effectiveness of pharmacist-structured review in reducing inappropriate PPI prescriptions and estimate the potential cost saving.

Methods: This non-randomised controlled study was conducted for 16 weeks at 17 government health clinics in Selangor, Malaysia. Eligible patients attending the outpatient pharmacies of intervention clinics were recruited consecutively and their consent was obtained. A structured review of PPIs was performed in which pharmacists identified patient demographics, indications and the length of PPI therapy using a PPI intervention form. Recommendations were discussed with physicians before prescription changes were made and documented. Moreover, standard management was conducted in the control clinics.
\end{abstract}

Results: A total of 568 patients with prescriptions containing PPIs were sampled, with a total of 284 patients being placed into the control and intervention groups, respectively. Compared to the control group, inappropriate PPI utilisation in the intervention group significantly decreased from 79.9 to $30.4 \%(\mathrm{p}<0.05)$. The changes to PPI prescriptions observed in the intervention group included: stop PPI therapy (30.8\%), step-down therapy $(22.9 \%)$, start substitution therapy $(15.9 \%)$ and no change (30.4\%). The physicians' acceptance rate for pharmacist intervention was $67.8 \%$. A $66.1 \%$ reduction in monthly PPI pill count and a $72.0 \%$ reduction in monthly medication expenditure (RM44.85/patient/year) were observed.

Conclusion: The pharmacist-structured review was effective in increasing appropriate PPI utilisation and led to substantial cost savings.

\section{Introduction}

Proton pump inhibitors (PPIs) suppress gastric acid secretion and effectively treat acid-related gastrointestinal disorders such as hypersecretory conditions, duodenal ulcers, gastric ulcers, gastroesophageal reflux disease (GERD), heartburn, upper gastrointestinal bleeding and Zollinger-Ellison syndrome, whilst also being useful in Helicobacter pylori eradication as well as stress ulcer- and drug-induced peptic ulcer prophylaxis. ${ }^{1,2}$ Their effectiveness has contributed to the overuse of PPIs, which has become a growing global concern., ${ }^{2,3}$ The overutilisation of PPIs in the ambulatory care setting often stems from the failure of the hospital to discontinue prophylaxis treatment before discharge, re-evaluate PPI therapy and consider on-demand and step-down therapy following discharge. ${ }^{4}$
Numerous studies have emerged with reports on the overutilisation of PPIs, claiming up to $68 \%$ inappropriate PPI indication among hospital inpatients in developed countries. ${ }^{4,5}$ An observational study conducted in 31 primary care settings in Germany found that $58 \%$ of patients were discharged from hospital without any clear indication of PPIs; however, PPIs were continued by primary care physicians for at least 1 month thereafter. ${ }^{6}$ Notably, inappropriate long-term PPI use can lead to poorer clinical outcomes. Patients who are on long-term PPI therapy are more susceptible to variations in the bioavailability of common medications, vitamin B12 deficiency, Clostridium difficile infections, communityacquired pneumonia as well as hip, wrist and spine fractures.? 
Patients who were prescribed long-term PPI use should be regularly reviewed to assess the need for continuation. ${ }^{8}$ Canadian Consensus Guidelines and Australian National Prescribing Guidelines recommend that PPIs should be discontinued after 4-8 weeks of initial treatment unless indicated for longterm treatment. If symptoms recur, the reintroduction of PPIs at the lowest dose and frequency necessary to control symptoms is recommended. ${ }^{9,10}$ Although treatment algorithms are useful for day-to-day practice, a systematic review recommended that the dosage and duration of a PPI treatment should be individualised to each patient's condition and setting. ${ }^{11}$ Notably, medication review has been useful in optimising patient medication utilisation especially for those with chronic conditions. ${ }^{12}$ In primary care, a pharmacistled medication review is an essential strategy that enables appropriate PPI use. Pharmacists can reduce the overuse of PPIs and their associated costs in primary care by conducting a standardised, guided intervention. ${ }^{13-15}$

Studies performed in hospitals within the states of Sarawak, Pahang and Selangor reported that $58.1,31$ and $46 \%$ of inpatients on PPI acid suppression treatment did not have appropriate indications, respectively. ${ }^{3,16,17}$ However, issues of PPI continuation and treatment review have not been comprehensively explored among primary care clinics in Malaysia. The overutilisation of PPIs in our healthcare setting has raised clinical and financial concerns among pharmacists. An average PPI usage of 17 months was observed, with an expenditure of RM91.36/patient/year.

Our study aimed to evaluate the effectiveness of pharmacist intervention on long-term PPI use by comparing the differences in appropriate prescribing and cost savings between the intervention and control groups.

\section{Methods}

Study Design and Setting

This was a multi-centre, non-randomised controlled study on the effectiveness of the pharmacist-structured review of PPI prescriptions in primary care. The study was conducted in 17 government primary healthcare clinics located in two different districts in Selangor State. These districts were purposely selected due to their comparable population numbers and sociodemographics. ${ }^{18}$ Nine clinics in the same district were chosen as intervention sites where the pharmacist- structured review of inappropriate PPI use was initiated by the district's standardised clinical management. The remaining eight clinics under the second district were assigned as control sites that had no such review.

Choosing clinics under the same district as control groups would ensure that the patients were naive to the intervention and any inappropriate PPI use was solely intervened via typical practice. A total of three clinics with no resident pharmacist were excluded from the study since the review of prescriptions by pharmacists was not possible. Another three clinics with no computerised prescription records were also excluded. Data collection was conducted for a 6-month period from November 2017 to April 2018.

\section{Sample Size Estimation}

The total population was estimated based on the PPI prescription records generated by the computerised dispensing systems (i.e., Pharmacy Information System (PhIS) and TelePrimary Care System (TPC)) implemented by all the clinics since 2015. Based on a previous study by $\mathrm{Oh}$ et al., the sample size was computed using the Two-Proportion Sample Size Calculation in EpiCalc2000 with 99\% CI, whilst an additional 10\% accounted for loss. To represent the total population of patients using PPIs, the intervention and control groups required a minimum of 266 samples each.

\section{Participants}

In both intervention and control clinics, consecutive patients attending outpatient pharmacies within the study period were identified by pharmacists at the screening counters. Randomisation or systematic sampling was not feasible in our subject recruitment since patients' attendance at the pharmacy counter was not predetermined. However, the control group was essentially equivalent to the intervention group based on known pre-intervention characteristics, which would eliminate unknown biases.

The inclusion criteria for both groups were adult patients (above 18 years old) and present with any type of new PPI prescription or refill prescription. The exclusion criteria were currently on Helicobacter pylori eradication therapy, terminally ill, currently undergoing chemotherapy or radiotherapy, waiting for referral appointments at other healthcare facilities and requiring less than 8 weeks of treatment. 
Additional exclusion criteria for patients in the intervention clinics were cognitive impairment, unable to communicate well and represented by another person to collect the medication.

\section{Study Instrument}

The pharmacist-structured review was conducted using a PPI intervention form (Appendix 1) in the intervention clinics. This form was adapted from National Prescribing Service Limited, Australia, 2009. ${ }^{11}$ The form was modified to suit our study objectives and evaluated before use by two senior pharmacists and one family medicine specialist (FMS) who were not otherwise involved in the study. The form and data collection workflow was piloted by involving eight respondents in one of the intervention clinics. Since the form was completed by trained pharmacists, there was no requirement for the content to be translated from English to other languages.

This form was used to extract the patient data, dose and indication of prescribed PPIs, length of PPI therapy and concurrently prescribed medication that may exacerbate gastrointestinal symptoms or interact with the PPIs. Patients' responses about their current PPI dose consumption and concurrent self-medication were also captured. The form was also used to record pharmacists' recommendations based on their structured review as well as changes made to the PPI prescription following consultations with the doctors.

In the intervention clinics, the research team provided two series of central training for pharmacists on how to recruit respondents, extract data from the prescriptions and patient medical records and how to complete the PPI intervention form. For items that need responses from the subjects, the pharmacists were required to read out all of the answer choices. They were also given continuous pharmacy education (CPE) on the rational use of PPIs based on clinical guidelines by a clinical pharmacist to standardise their assessment of the appropriateness of PPI prescriptions. Training sessions were repeated by the trained pharmacists at individual clinics using a standardised training slide presentation. An inhouse PPI counselling guideline and PPI patient information leaflet (PIL) were provided by the Clinical Pharmacy Unit and made available in the pharmacies of the intervention clinics.

Intervention

In the intervention clinics, pharmacists at the screening counter attached the patient information, consent form and PPI intervention form to each selected prescription. All eligible and consenting patients were interviewed and counselled at the pharmacy counter during medication dispensing by another pharmacist assigned to the dispensing counter. Structured reviews of PPI prescriptions were individualised and guided step by step as the pharmacists completed the information required in the PPI intervention form.

For patients with inappropriate PPIs and those who were non-compliant to PPIs and symptom-free, pharmacists made recommendations to either reduce the dose, change PPI use to an as-needed basis (PRN) or every other day (EOD) dosing, or stop PPI with or without $\mathrm{H}_{2}$-antagonist therapy initiation. Pharmacists' interventions and recommendations were communicated to the physicians via telephone calls. Changes in the medication regime were made upon the physicians' agreement and recorded. Since pharmacists' review notes were attached to patients' clinic appointment cards, the intervention will be noted during subsequent clinic visits.

Counselling on the changes agreed upon by physicians regarding the PPI regime, utilisation of $\mathrm{H}_{2}$-antagonist therapy or antacids on a PRN basis, and lifestyle changes was provided to patients during medication dispensing. PILs were disseminated to the patients at this point. For patients with appropriate PPI use, counselling on medication adherence, drugdrug interaction, the monitoring of adverse reactions and alarm symptoms was given.

In the control clinics, no structured review, prescription intervention or counselling on PPIs were performed.

\section{Data Collection}

Data for the intervention group was extracted from the completed PPI intervention forms from all clinics by two researchers. Agreement on data interpretation from both researchers was maintained. For the control group, data were extracted from prescriptions as well as patient medical and supply records. In both groups, the PPI regime for each patient was captured at baseline and the changes in subsequent prescriptions were traced via the computerised prescription record 16 weeks after the initial recruitment. 
Patients' PPI indication and duration of therapy were two important parameters in evaluating its appropriateness. For both groups, PPI indication had to be identified from patients' medical records or referral letters if it was unavailable from the prescriptions. To identify the treatment duration, the PPI start date was taken from the discharge date stated in the referral letter from hospitals. If the PPIs were started in the health clinics, the date of the first prescription was considered the PPI start date.

\section{Outcome Measurement}

There were three main outcome measures in the intervention and control clinics: 1) the rate of inappropriate PPI prescriptions (analysed using descriptive analyses); 2) therapy changes 16 weeks after initial recruitment; 3) potential medication cost savings in terms of the pill count per month and average cost per month of PPI usage. According to the Canadian Family Physicians Evidence-based Clinical Practice Guideline on Deprescribing PPI 2017, inappropriate PPI utilisation is defined as unclear, unknown and undocumented indication and duration of treatment. ${ }^{11}$ PPI treatments initiated for a short course of 4-8 weeks are regarded as long-term treatment if the duration extends beyond 8 weeks. For the first outcome, PPI prescriptions were classified as inappropriate when their utilisation was not indicated for long-term treatment in accordance with this guideline.
Data Analyses

Data analysis was performed using the Statistical Package for Social Science (SPSS) version 21 and Microsoft Excel 2011. All descriptive analyses were reported in frequencies, means and standard deviations with 95\% confidence intervals. Comparative analyses were conducted using the appropriate binomial test, t-test or chi-squared test since the age, treatment regime and duration of PPI treatment in control and intervention groups were normally distributed. Confidence levels were set at $95 \%$ and a $p$-value of $<0.05$ was regarded as statistically significant.

\section{Ethical Considerations}

This study was registered under the National Medical Research Register (NMRR) with registration number NMRR-17-3097-37220, approved by the Malaysian Research Ethical Committee (MREC) and allowed by relevant local and national authorities. All patient details were treated as private and confidential and kept under the strict control of the researchers only.

\section{Results}

A total of 290 eligible patients were initially enrolled into the intervention and control groups, respectively. However, 6 patients were excluded (3 per group) due to the unavailability of subsequent prescriptions, resulting in a total of 284 patients per group.

Table 1. Baseline characteristics of the study population $(\mathrm{N}=568)$ of 17 primary care clinics.

\begin{tabular}{|c|c|c|c|}
\hline Characteristics & Control group ( $n=284)$ & Intervention group ( $\mathrm{n}=\mathbf{2 8 4})$ & p-value \\
\hline Age (years) $*($ Mean $\pm S D)$ & $63.4 \pm 12.2$ & $63.8 \pm 11.6$ & 0.686 \\
\hline $\begin{array}{l}\text { Gender** } \\
\text { Female } \\
\text { Male }\end{array}$ & $\begin{array}{l}153(53.9) \\
131(46.1)\end{array}$ & $\begin{array}{l}155(54.6) \\
129(45.4)\end{array}$ & 0.917 \\
\hline $\begin{array}{l}\text { Race** } \\
\text { Malay } \\
\text { Chinese } \\
\text { Indian } \\
\text { Other }\end{array}$ & $\begin{array}{c}136(47.9) \\
76(26.8) \\
66(23.2) \\
6(2.1)\end{array}$ & $\begin{array}{c}93(32.7) \\
79(27.8) \\
108(38.0) \\
4(1.4)\end{array}$ & $<0.001$ \\
\hline $\begin{array}{l}\text { Type of PPI** } \\
\text { Omeprazole } \\
\text { Esomeprazole } \\
\text { Pantoprazole }\end{array}$ & $\begin{array}{c}180(63.3 \%) \\
1(0.7 \%) \\
103(36.3 \%)\end{array}$ & $\begin{array}{c}234(82.4 \%) \\
8(2.9 \%) \\
42(14.7 \%)\end{array}$ & 0.103 \\
\hline $\begin{array}{l}\text { Frequency of PPI }{ }^{* *} \text { Twice } \\
\text { daily (BD) } \\
\text { Once daily (OD) } \\
\text { Every other day (EOD) } \\
\text { When necessary (PRN) }\end{array}$ & $\begin{array}{c}5(1.8 \%) \\
273(96.1 \%) \\
1(0.4 \%) \\
5(1.8 \%)\end{array}$ & $\begin{array}{c}5(1.6 \%) \\
260(91.5 \%) \\
0(0.0 \%) \\
19(6.9 \%) \\
\end{array}$ & 0.025 \\
\hline $\begin{array}{l}\text { Duration of PPI therapy* } \\
* \text { Mean } \pm \text { SD } \\
\text { *Range (month) }\end{array}$ & $\begin{array}{c}14.1 \pm 11.5 \\
3-62\end{array}$ & $\begin{array}{c}16.9 \pm 11.1 \\
3-77\end{array}$ & 0.003 \\
\hline
\end{tabular}

All values are reported as $n(\%)$ unless otherwise stated $(*)$.

* Independent T-Test, $t$.

** Chi-square test for independence, $x^{2}$. 
Most patients were Malay females with a mean age of 63 years. There were no statistically significant differences between the two groups with respect to mean age and gender distribution. However, both groups had different ethnic distributions. Although both groups have a similar PPI prescription trend in terms of PPI choice, no similarities were observed in PPI frequencies and treatment duration at baseline. Differences in PPI prescribing patterns were expected since the intervention and control clinics were under two different districts with different practices and prescribing preferences.

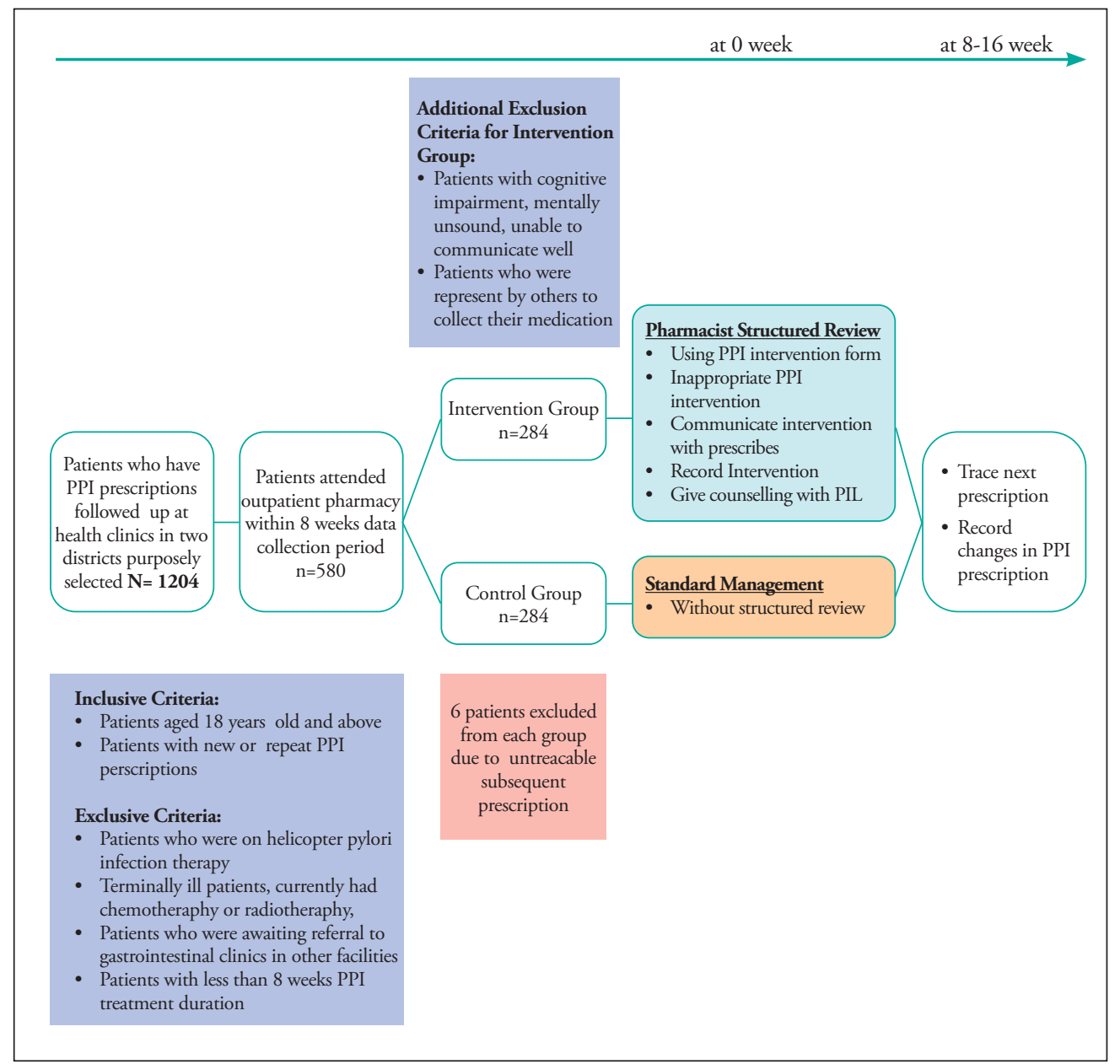

Figure 1. Flowchart describing the subject recruitment and study process.

Table 2. Clinical indications for PPI therapy.

\begin{tabular}{|l|c|c|} 
Clinical indication & $\begin{array}{c}\text { Control group } \\
(\mathbf{n}=\mathbf{2 8 4})\end{array}$ & $\begin{array}{c}\text { Intervention group } \\
(\mathbf{n}=\mathbf{2 8 4})\end{array}$ \\
\hline NOT indicated for long-term use (inappropriate PPI continuation)
\end{tabular}

\begin{tabular}{l|c|c}
\hline GERD/reflux/dyspepsia/heartburn & $19(6.7 \%)$ & $83(29.2 \%)$ \\
\hline Mild to moderate oesophagitis & $1(0.4 \%)$ & $8(2.8 \%)$ \\
\hline $\begin{array}{l}\text { Peptic ulcers (including Non-steroidal anti-inflammatory } \\
\text { drugs(NSAIDs) or H. pylori-induced ulcers) }\end{array}$ & $7(2.5 \%)$ & $15(5.3 \%)$ \\
\hline H. pylori eradication & $1(0.4 \%)$ & $3(1.0 \%)$ \\
\hline Unknown & $171(60.2 \%)$ & $105(36.9 \%)$ \\
\hline Other & $26(9.2 \%)$ & $13(4.6 \%)$ \\
\hline Total & $\mathbf{2 2 5 ( 7 9 . 2 \% )}$ & $\mathbf{2 2 7}(\mathbf{7 9 . 9} \%)$ \\
\hline
\end{tabular}




\begin{tabular}{l|c|c} 
Clinical indication & $\begin{array}{c}\text { Control group } \\
(\mathbf{n}=\mathbf{2 8 4})\end{array}$ & $\begin{array}{c}\text { Intervention group } \\
(\mathbf{n}=\mathbf{2 8 4})\end{array}$ \\
\hline Indicated for long-term use & $59(20.8 \%)$ & $54(19.0 \%)$ \\
\hline Prophylaxis of drug-induced dyspepsia/ulceration & $0(0.0 \%)$ & $2(0.7 \%)$ \\
\hline Zollinger-Ellison syndrome & $0(0.0 \%)$ & $0(0.0 \%)$ \\
\hline Barrett's oesophagus & $0(0.0 \%)$ & $1(0.4 \%)$ \\
\hline Severe oesophagitis & $0(0.0 \%)$ & $0(0.0 \%)$ \\
\hline Strictures, scleroderma & $\mathbf{5 9 ( 2 0 . 8 \% )}$ & $\mathbf{5 7 ( 2 0 . 0 \% )}$ \\
\hline Total & &
\end{tabular}

Table 2 presents the clinical indications for PPI therapy in the study population. At baseline, similar numbers of patients with inappropriate PPI prescriptions were observed in both groups $(\mathrm{n}=227,79.9 \%$ in the intervention group and $\mathrm{n}=225,79.2 \%$ in the control group). In both groups, an unknown reason for the continuation of PPIs was observed in nearly half of the patients $(n=276 / 568,48.6 \%)$.

Primary Outcome: Rate of Inappropriate PPI Prescriptions

After the 16-week intervention period, 86 patients (30.3\%) continued with PPI prescriptions. This represents a statistically significant reduction $(\mathrm{p}<0.05)$ of inappropriate PPI use with the intervention (Figure 2). In the control group, 219 patients (77.1\%) continued with inappropriate PPI prescriptions and followed standard management at the care setting at the end of the study period.

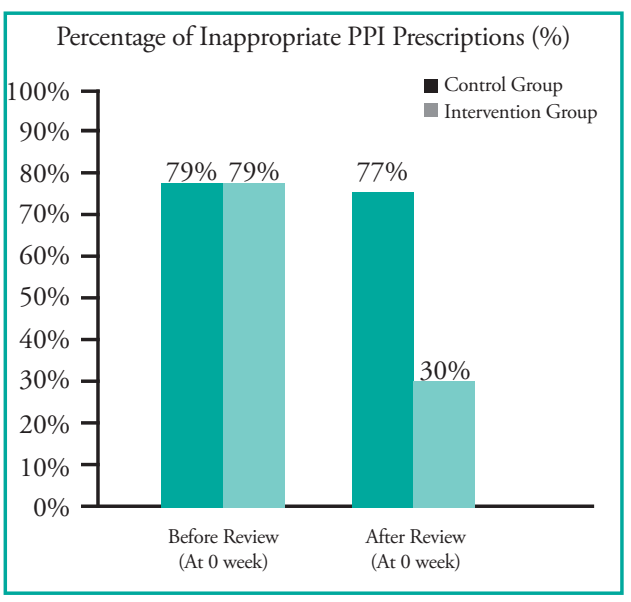

Figure 2. Inappropriate PPI prescription reduction with and without pharmacist intervention. ${ }^{*} \mathrm{p}<0.05$, post-intervention, chisquared test.

Secondary Outcome: Therapy Changes In the intervention group, pharmacist recommendations were made via phone calls to the physicians of 227 patients upon identification of inappropriate PPI usage. Changes were made to 198 prescriptions (Table 3), resulting in an $87.2 \%$ acceptance rate for pharmacist recommendations. In the control group, no changes were observed for most prescriptions.

Table 3. Changes to PPI prescriptions after review.

\begin{tabular}{l|c|c}
$\begin{array}{l}\text { Changes to PPI } \\
\text { prescriptions }\end{array}$ & $\begin{array}{c}\text { Intervention } \\
\text { group } \\
(\mathbf{N}=\mathbf{2 8 4})\end{array}$ & $\begin{array}{c}\text { Control } \\
\text { group } \\
(\mathbf{N}=284)\end{array}$ \\
\hline $\begin{array}{l}\text { No changes to } \\
\text { prescriptions }\end{array}$ & $86(30.4 \%)$ & $265(93.3 \%)$ \\
\hline $\begin{array}{l}\text { Stop PPI } \\
\text { therapy }\end{array}$ & $88(30.8 \%)$ & $6(2.2 \%)$ \\
\hline $\begin{array}{l}\text { Step-down } \\
\text { therapy }\end{array}$ & $65(22.9 \%)$ & $1(0.4 \%)$ \\
\hline $\begin{array}{l}\text { Substitution } \\
\text { therapy }\end{array}$ & $45(15.9 \%)$ & $12(4.0 \%)$ \\
\hline
\end{tabular}

Tertiary Outcome: Potential Medication Cost Saving

In the intervention group, a significant reduction $(66.1 \%)$ in the monthly PPI pill count and a $72.0 \%$ reduction in monthly medication expenditure (RM44.85/patient/ year) were observed.

Table 4. Monthly pill count and monthly pill cost saved after 16 weeks of intervention.

\begin{tabular}{l|c|c}
\hline $\begin{array}{l}\text { Intervention } \\
\text { group }\end{array}$ & $\begin{array}{c}\text { Monthly } \\
\text { pill count } \\
\text { (tablets) }\end{array}$ & $\begin{array}{c}\text { Monthly pill } \\
\text { cost (RM) }\end{array}$ \\
\hline Before review & 13080 & 6184.05 \\
\hline After review & 4435 & 1733.90 \\
\hline Difference & $\begin{array}{c}8645 \\
(66.1 \%)\end{array}$ & $\begin{array}{l}4450.15 \\
(72.0 \%)\end{array}$ \\
\hline
\end{tabular}

*Price per pill: Esomeprazole $=$ RM 2.35/tab, Omeprazole $=\mathrm{RM} 0.44 / \mathrm{tab}$, Pantoprazole $=\mathrm{RM}$ $0.11 / \mathrm{tab}$

* Ministry of Health approved product price list and central contract (updated October 2017). 


\section{Other Drug-Related Problems}

In the intervention group, patients PPI compliance was reported in the PPI intervention form, where pharmacists asked each patient how they currently take their PPIs. A total of 114 (40.1\%) patients reported their non-compliance towards PPIs. Out of these patients, 35 (30.7\%) were not taking them, $73(64.0 \%)$ were taking them when necessary (PRN) and $6(5.3 \%)$ were taking them on an EOD basis without any consultation with healthcare providers. This accounted for an estimated 4,755 unconsumed pills per month with an unused drug wastage of RM 8,156.85 per month.
This study identified four drug classes that can exacerbate or induce dyspepsia or ulceration that were taken concurrently with PPIs by 141 (49.6\%) patients in the intervention group (see Figure 3). A total of $30(10.6 \%)$ patients took medications that alter gastric pH (e.g., antacids and H2antagonists) concurrently with PPI for their symptomatic control, which suggests the ineffectiveness of the PPIs they were taking. Physicians were notified of these cases for review during patients' next appointment, where they may need to be seen by an FMS.

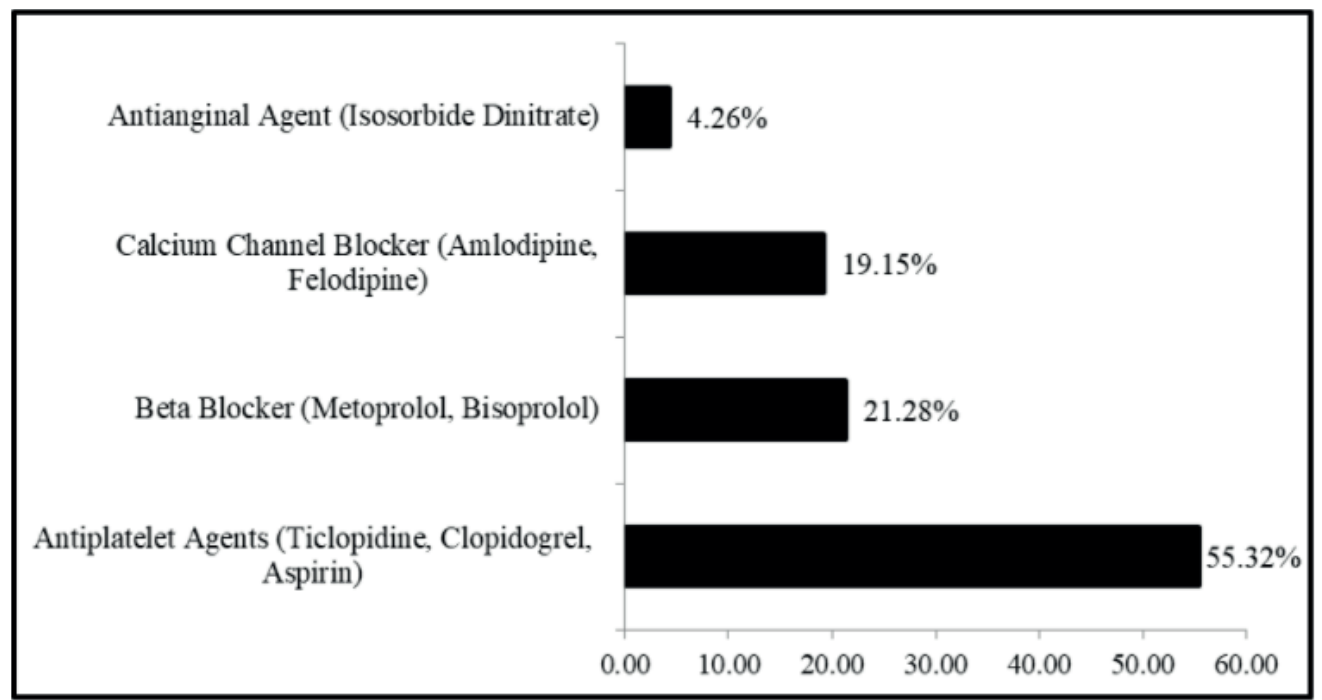

Figure 3. Concomitant use of medications that induce/exacerbate dyspepsia or ulceration.

\section{Discussion}

This study demonstrated that pharmaciststructured review effectively reduced the number of inappropriate PPI prescriptions whilst leading to therapy optimisation and substantial medication cost saving. Notably, drug-related problems (DRPs) related to medication non-compliance and drug-drug interaction can be concurrently addressed during such reviews.

Inappropriate PPI prescriptions were apparent in the majority $(79 \%)$ of patients in public primary care clinics, while studies performed in Malaysian tertiary hospitals showed that 1 to $58 \%$ of the prophylactic PPIs prescribed were unnecessary. ${ }^{3,16,17}$ With pharmacist intervention, the appropriateness of PPI prescriptions was significantly improved by $49.5 \%$, with the desired outcomes being on par with those of another similar study
$(53.1 \%)^{14}$ adopting a similar framework to increase the appropriate use of PPIs.

It was also observed that the rate of appropriate PPI use increased as the intervention period progressed, which suggests the need for greater awareness of this intervention as well as structured reviews of PPI use among pharmacists and physicians. The effectiveness of structured intervention tools for pharmacists was evident since there were no changes in the appropriateness of PPI use observed in the control clinics, with $77.1 \%$ of PPI users still having inappropriate PPI prescriptions at the end of the study period.

In both the intervention and control groups, 92.6\% of PPI prescriptions were initiated by tertiary care hospitals. Patients were then referred to health clinics for the continuation 
of care. At baseline, patients had a mean PPI treatment duration of more than 1 year. These findings highlight the need for medication review following hospital discharge and raise concerns regarding potential side effects and higher health care costs.

In the context of this study, the continuation of PPI after hospital discharge may not be necessary since 4-8 weeks of PPI treatment had been completed through hospital inpatient and post-discharge outpatient prescriptions. Several studies have also shown that PPIs are often overused in the ambulatory care setting due to a lack of documented indications leading to the inappropriate and chronic use of PPIs. ${ }^{2,12}$

The pharmacists' recommendations for PPIs therapy changes achieved an $87.2 \%$ acceptance rate from the physicians, which is consistent with the results reported in another study (89.4\%). ${ }^{14}$ We did not enquire about the reasons for the remaining pharmacists' recommendations not being accepted. However, based on pharmacists' observations, physicians may have made their own clinical judgments based on the individual patient since PPI tapering down may not be suitable due to factors such as age, the seriousness of the disease or the unwillingness of the patient to changes in therapy. Therefore, pharmacist recommendations may not be the only factor causing physicians to change PPI therapies. ${ }^{19,20}$

Pharmacist intervention in PPI use profoundly contributed to reducing medication expenses by up to $72 \%$. This was also observed in a previous study, which noted a $72.6 \%$ cost reduction. ${ }^{14}$ However, our study did not further investigate the costs incurred due to substitution therapy, treatment for rebound gastrointestinal symptoms and the re-initiation of PPIs. The cost of hospital admissions or referrals were also not investigated.

Pharmacist-structured reviews using a guided checklist may also highlight therapeutic issues such as drug-drug interaction, contraindication and non-compliance issues in patients. Highlighting these DRPs for physicians may further reduce the medication list, optimise the treatment of other underlying chronic diseases and improve patients' medication compliance. Notably, this would require another study outlining the different objectives of this study.

The current clinical practice guideline available in Malaysia does not specifically mention the deprescribing of PPI therapy.20 In our setting, there is no standard practice for PPI deprescription, which may have limited physicians' acceptance of pharmacists' recommendations. This has led to our interest in embarking on a future collaboration with physicians and FMSs to implement a PPI deprescribing algorithm.

A multi-disciplinary approach to improving the appropriateness of PPIs utilisation can be advocated based on several published clinical guidelines. ${ }^{9,10,13}$ It is important to routinely review this group of patients to ensure clinical and financial benefits for the respective stakeholders. The implementation of a PPI deprescribing algorithm is highly recommended to standardise the management of patients who are newly discharged or newly initiated with PPIs.

\section{Study Limitations}

We experienced difficulties in obtaining complete patient medication histories and indications for PPI from the hospital referral letters in more than half of all subjects. This highlighted the importance of properly documenting referrals to primary care since confirmed diagnoses indicating long-term PPI use cannot be obtained via procedures or tests in a primary care setting. ${ }^{14}$

There was also a lack of information on how long the patients had been on PPI treatment and how long the treatment should be continued. As a result, the PPI start date was assumed to be the date of referral to the primary care level. Thus, therapy durations may be under-reported.

We did not follow the subjects after the intervention in PPI therapy to note any changes in patients' clinical outcomes. Therefore, whether or not there was any reduction in the adverse effects of longterm PPI use remains unknown. It would be valuable for future studies to measure the reduction of associated incidences and clinical outcomes after deprescription. Moreover, this study did not consider whether the structured review potentially reduced the bioavailability of other concomitant medications that interact with PPIs.

Since this was a non-randomised controlled study, the results should be interpreted with caution since potential biases may have 
affected the analyses. We suggest that it would be valuable for future studies to be conducted with the rigorous standards of a randomised clinical trial to provide a higher level of confidence in the results.

\section{Conclusion}

The inclusion of pharmacists in a structured medication review framework demonstrated significant impacts on PPI use when compared to current standard management without pharmacist involvement. This study shows that judicious prescribing and patient care can potentially lead to medication cost savings.

\section{Acknowledgements}

We would like to thank the Director-General of Health, Ministry of Health Malaysia for the study approval and National Medical Research Registration Identity (NMRR-173097-37220) required to conduct this study. We also thank Dr Goh Pik Pin from the National Clinical Research Centre (NCRC) for providing us with the time and resources necessary to work on the publication of this paper.

\section{How does this paper make a difference to general practice?}

- This paper highlights the effectiveness of a pharmacist-structured review in detecting and reducing the inappropriate use of proton pump inhibitors (PPIs).

- Inappropriate PPI use significantly decreased in the intervention group when compared to the control group.

- The pharmacist-structured review protocol implemented in this study can be replicated in other health care settings.

- This study provides baseline findings for future work in developing and implementing a PPI deprescribing algorithm in the primary care setting.

\section{References}

1. Scarpignato C, Gatta L, Zullo A, et al. Effective and safe proton pump inhibitor therapy in acid-related diseases - A position paper addressing benefits and potential harms of acid suppression. BMC Med. 2016;14(1):179.

2. Heidelbaugh JJ, Kim AH, Chang R, et al. Overutilization of proton-pump inhibitors: What the clinician needs to know. Therap $A d v$ Gastroenterol. 2012;5(4):219-32.

3. Oh AL, Tan AG, Phan HS, et al. Indication of acid suppression therapy and predictors for the prophylactic use of proton-pump inhibitors vs. histamine-2 receptor antagonists in a Malaysian tertiary hospital. Pharm Pract. 2015;13(3):633.

4. Parente F, Cucino C, Gallus S, et al. Hospital use of acid-suppressive medications and its fall-out on prescribing in general practice: A 1-month survey. Aliment Pharmacol Ther. 2003;17(12):1503-6.
5. Mat Saad AZ, Collins N, Lobo MM, et al. Proton pump inhibitors: A survey of prescribing in an Irish general hospital. Int $J$ Clin Pract. 2005;59(1):31-4.

6. Ahrens, D, Behrens G, Himmel W, et al. Appropriateness of proton pump inhibitor recommendations at hospital discharge and continuation in primary care. Int J Clin Pract. 2012;66(8):767-73

7. Nishtala PS, Soo L. Proton pump inhibitors utilisation in older people in New Zealand from 2005 to 2013. Intern Med J. 2015;5(6):624-9.

8. Grant K, Al-Adhami N, Tordoff J, et al. Continuation of proton pump inhibitors from hospital to community. Pharmacy World and Science. 2006;28(4):183-93.

9. Durand C, Willett KC, Desilets AR. Proton pump inhibitor use in hospitalized patients: Clinical medicine insights. Gastroenterology. 2012;65-76.
10. Farrell B, Pottie K, Thompson W, et al. Deprescribing proton pump inhibitors: Evidence-based clinical practice guideline. Can Fam Physician. 2017;63(5):354-64.

11. National Prescribing Service Limited. Pharmacy Practice Review: A Counselling and Action Resource. Quality Use of Prescription PPIs. New South Wales: Australian Government Department of Health and Ageing; 2009.

12. Hampson N, Ottey D. Pharmacist-led reviews can help patients and practices. Med Optim. 2015;26(7):15-7.

13. Bundeff AW, Zaiken K. Impact of clinical pharmacists' recommendations on a proton pump inhibitor taper protocol in an ambulatory care practice. J Manag Care Pharm. 2013;19(4):325-44.

14. Xin C, Dong Z, Lin M, et al. The impact of pharmaceutical interventions on the rational use of proton pump inhibitors in a Chinese hospital. Patient Preference Adherence.2018(12):21-6. 
15. Velasquez KL, Alvarez AM, Davis PN. A pharmacist-led medication use evaluation of proton-pump inhibitors. J Pharm Pharmacol. 2017;(5)864-868.

16. Elnaem MH, Nik Mohamad MH, Nazar $\mathrm{AH}$, et al. Evaluation of proton pump inhibitors prescribing among non-critically ill hospitalized patients in a Malaysian tertiary hospital. J Appl Pharm Sci. 2017;(7):77-83.
17. Fah, T., Jun, T., Yan, P., \& Yu, C. Appropriateness of proton pump inhibitors prescription in patients admitted to a Malaysian tertiary hospital. Int J Public Health Res. 2019;9(1):1043-50.

18. Laporan Tinjauan Kajian Rancangan Struktur Negeri Selangor 2035. A2: Penduduk dan Sumber. Jabatan Perancangan Bandar dan Desa Negeri Selangor; 2019.
19. Michal J, Henry T, Street C. Impact of a pharmacist-driven protocol to decrease proton pump inhibitor use in non-intensive care hospitalized adults. AJHP. 2016;73(17): S126-32.

20. Ministry of Health Malaysia. Clinical Practice Guidelines: Management of Non-Variceal Upper Gastrointestinal Bleeding. Academy of Medicine Malaysia; 2003. 